\title{
Fluctuations of conserved charges within a Hadron Resonance Gas approach: chemical freeze-out conditions from net-charge and net-proton fluctuations
}

\author{
V. Mantovani Sarti ${ }^{1}$,a, P. Alba ${ }^{1}$, W. Alberico ${ }^{1}$, R. Bellwied ${ }^{2}$, M. Bluhm ${ }^{3}$, M. Nahrgang ${ }^{4}$, C. Ratti ${ }^{2}$ \\ ${ }^{1}$ Department of Physics University of Turin, ${ }^{2}$ University of Houston, ${ }^{3}$ North Carolina State University, \\ ${ }^{4}$ Duke University
}

\begin{abstract}
We present an analysis of fluctuations of conserved charges, as baryon number $B$ and electric charge $Q$, using the Hadron Resonance Gas (HRG) model. The study of such observables within a theoretical approach and the comparison to experimental data on the moments of the multiplicity distributions of specific particles represents a useful tool to extract the chemical freeze-out parameters, temperature $T$ and baryo-chemical potential $\mu_{B}$. In particular we calculate ratios of higher-order susceptibilities for net-protons and net-charge and we compare our results to the latest data from STAR collaboration. The effect of resonance decays and kinematic cuts applied in the experimental analysis in transverse momentum, rapidity and pseudo-rapidity are included in the calculation along with the randomization of nucleons occurring in the hadronic phase.
\end{abstract}

\section{Introduction}

The understanding and the description of the phase diagram of nuclear matter under extreme conditions, high temperature $T$ and/or baryo-chemical potential $\mu_{B}$, represent major theoretical and experimental challenges. Heavy Ion Collisions (HICs) at experimental facilities provide a unique tool to explore a wide area of this phase diagram, from the high baryo-chemical region covered by AGS, SPS, Beam Energy Scan at RHIC and the future NICA and FAIR facilities to the high temperature regime covered by top energies at RHIC and LHC.

The dynamical evolution of a HIC proceeds from a system of deconfined quarks and gluons, the socalled Quark-Gluon Plasma (QGP), formed at the early stages of the collision, which cools down to a final stage described by hadronic degrees of freedom which undergo elastic and inelastic scatterings. In particular when inelastic collisions among hadrons stop, the hadro-chemistry and the abundances of particle species are fixed. This stage is called chemical freeze-out and is entirely determined by the corresponding values of temperature $T_{c h}$ and baryo-chemical potential $\mu_{B, c h}$.

So far, these freeze-out parameters have been extracted by means of Statistical Hadronization Models (SHMs) which fitted the data on particle yields and ratios in a wide range of energies, from AGS to LHC [1-5].

Recently the study of fluctuations of conserved charges in Lattice QCD (LQCD) [6-9] and in a HRG

\footnotetext{
ae-mail: mantovan@to.infn.it
} 
approach [10-15] has been proposed as another tool to obtain the values of the freeze-out parameters and experimental data for these observables have been published by the STAR collaboration [16, 17]. The description of the low temperature regime of strongly interacting matter within a HRG model shows a nice agreement with LQCD calculations up to $T \approx 170 \mathrm{MeV}$, near the crossover region $[18,19]$. The study of fluctuations of conserved charges in a HRG approach can overcome some of the limitations of LQCD calculations since it allows us to include the experimental acceptance cuts on transverse momentum, rapidity and pseudo-rapidity to study observables for individual particle species and to explore the finite $\mu_{B}$ regime.

In this paper, we evaluate ratios of susceptibilities for net-protons and net-electric charge fluctuations in a HRG model by including the effect of resonance decays. By comparing our results to data from the STAR collaboration, we extract a chemical freeze-out parametrization in a large range of collision energies.

\section{The Hadron Resonance Gas model}

The hadronic stage occuring during the evolution of a HIC is described by means of the HRG model, namely by considering this phase as a gas of non-interacting hadrons and resonances in thermal and chemical equilibrium.

The main ingredient from which we derive all the thermodynamical observables is the logarithm of the grand-canonical partition function of particle $i$ :

$$
\ln \mathcal{Z}_{i}\left(T, \mu_{i}, V\right)= \pm \frac{V g_{i}}{2 \pi^{2}} \int_{0}^{\infty} p^{2} d p \ln \left[1 \pm \exp \left(-\frac{\sqrt{p^{2}+M_{i}^{2}}-\mu_{i}}{T}\right)\right]
$$

where $+(-)$ holds for fermions (bosons) and $V$ is the volume. The properties of the hadrons are included in the degeneracy factor $g_{i}$, in the mass $M_{i}$ and in the chemical potential $\mu_{i}=B_{i} \mu_{B}+Q_{i} \mu_{Q}+$ $S_{i} \mu_{S}$, where $B_{i}, Q_{i}$ and $S_{i}$ are the baryon, electric and strange charge of the particle while $\mu_{B}, \mu_{Q}$ and $\mu_{S}$ represent the chemical potentials associated to them.

Pressure $P$ is obtained from Eq. (1) by the relation $P=\frac{T}{V} \sum_{i} \ln \mathcal{Z}_{i}$, where the index $i$ runs over the hadron spectrum and the particle number density $n_{i}=\left(\frac{\partial P}{\partial \mu_{i}}\right)_{T}$ is given by:

$$
n_{i}\left(T, \mu_{i}\right)=\frac{g_{i}}{2 \pi^{2}} \int_{0}^{\infty} p^{2} d p \frac{1}{ \pm 1+\exp \left(-\left(\sqrt{p^{2}+M_{i}^{2}}-\mu_{i}\right) / T\right)}
$$

so that the densities associated to the conserved charges $X=B, S, Q$ become $n_{X}=\sum_{X} X_{i} n_{i}$. At the experiment the chemical potentials $\mu_{B}, \mu_{Q}$ and $\mu_{S}$ are not independent but the initial conditions occurring at the collision provide the following costraints:

$$
\begin{aligned}
& n_{S}\left(T, \mu_{B}, \mu_{Q}, \mu_{S}\right)=0 \\
& n_{Q}\left(T, \mu_{B}, \mu_{Q}, \mu_{S}\right)=\frac{Z}{A} n_{B}\left(T, \mu_{B}, \mu_{Q}, \mu_{S}\right), \frac{Z}{A}=0.4
\end{aligned}
$$

corresponding to the absence of initial content of net-strangeness and to the isospin asymmetry in the colliding nuclei given by the ratio of protons to nucleons.

The chemical equilibrium among hadrons is assumed at the transition temperature $T_{c}$ but, as the expansion of the fireball proceeds, the inelastic scattering rate is not high enough to provide a full 
chemical equilibrium at the hadronic stage before chemical freeze-out. For temperatures $T \leq T_{c h} \leq T_{c}$ hadronic resonance decays and regeneration processes continue to occur leading to hadronic matter in a partial chemical equilibrium [20]. The multiplicity of hadrons stable against strong decays is frozen at the chemical freeze-out and the inclusion of resonance decays modifies the final number of particles produced:

$$
\hat{N}_{i}=N_{i}+\sum_{R} N_{R}<n_{i}>_{R}
$$

where the sum runs over all the resonances $R$ decaying into $i, N_{i}$ and $N_{R}$ represent the primordial abundance of particles $R$ and $i$, and $\left\langle n_{i}>_{R}\right.$ is the average number of $i$ produced by the decay of resonances $R$.

The ensemble of stable particles, which we consider, is $\pi^{ \pm}, \pi^{0}, K^{ \pm}, K^{0}, \bar{K}^{0}$ and $p, n, \Lambda, \Sigma^{ \pm}, \Xi^{0,-}, \Omega$ (along with their corresponding anti-particles), while the hadron spectrum of resonances contains states up to a mass of $2 \mathrm{GeV}$, listed in the Particle Data Group [21, 22].

In order to make connection to experimental data which cover a finite phase space region, we also include the experimental kinematic cuts on transverse momentum, rapidity and pseudo-rapidity which modifies the thermodynamical integrals in the HRG calculations [10].

\subsection{Fluctuations of conserved charges}

The susceptibilities of conserved charges $B, Q, S$ represent a tool to probe the hadronic medium for the fluctuations of such conserved quantities in HICs and they are defined as:

$$
\chi_{l m n}^{B Q S}=\frac{\partial^{l+m+n} P / T^{4}}{\partial\left(\mu_{B} / T\right)^{l} \partial\left(\mu_{Q} / T\right)^{m} \partial\left(\mu_{S} / T\right)^{n}} .
$$

These quantities are strictly connected to the moments of multiplicity distributions experimentally available:

$$
\begin{array}{lr}
\text { mean : } \mathrm{M}=\langle\mathrm{N}\rangle=\mathrm{VT}^{3} \chi_{1}, & \text { variance }: \sigma^{2}=\left\langle(\delta \mathrm{N})^{2}\right\rangle=\mathrm{VT}^{3} \chi_{2}, \\
\text { skewness : } \mathrm{S}=\frac{\left\langle(\delta \mathrm{N})^{3}\right\rangle}{\sigma^{3}}=\frac{\mathrm{VT}^{3} \chi_{3}}{\left(\mathrm{VT}^{3} \chi_{2}\right)^{3 / 2}}, \quad \text { kurtosis : } \kappa=\frac{\left\langle(\delta \mathrm{N})^{4}\right\rangle}{\sigma^{4}}-3=\frac{\mathrm{VT}^{3} \chi_{4}}{\left(\mathrm{VT}^{3} \chi_{2}\right)^{2}}
\end{array}
$$

In order to not consider the volume, the following volume-independent ratios are usually considered:

$$
\begin{aligned}
& \frac{\sigma^{2}}{M}=\frac{\chi_{2}}{\chi_{1}}, S \sigma=\frac{\chi_{3}}{\chi_{2}}, \\
& \kappa \sigma^{2}=\frac{\chi_{4}}{\chi_{2}}, \frac{S \sigma^{3}}{M}=\frac{\chi_{3}}{\chi_{1}}
\end{aligned}
$$

The direct comparison between our HRG model calculations and efficiency corrected data from STAR collaboration on net-protons $\left(N_{n e t-p}=N_{p}-N_{\bar{p}}\right)$ and net-charge $\left(N_{\text {net-q }}=N_{+}-N_{-}\right)$ fluctuations $[16,17]$ follows from some preliminary considerations. First of all, the fluctuations of conserved charges come from the fact that in the experiment only a finite range of the phase space is accessible due to acceptance cuts and, as already mentioned in the previous section, such kinematic cuts have been taken into account in the HRG calculations. Moreover, for the net-proton case we are considering only a subset of particles which contribute to the baryon charge and we thus need a relation between fluctuations of a conserved charge such as $B$ and fluctuations of net-quantities such as net-protons. A theoretical scheme to provide these relations is given by Kitazawa and Asakawa 


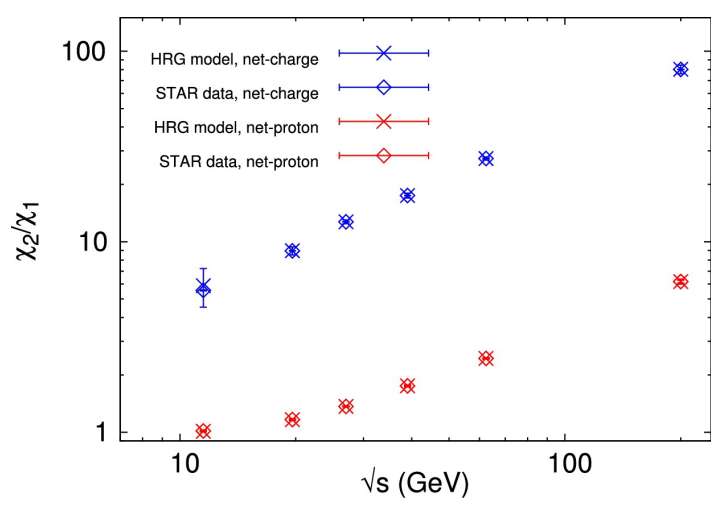

Figure 1: Results on the comparison of the ratio $\chi_{2} / \chi_{1}$ in the HRG model and most central experimental data from STAR.

(KA) in [23, 24] and it has been properly included in our calculations [25]. The net-electric charge fluctuations are not affected by this issue since data and our calculations are based on the contribution from pions, kaons and protons which constitute the most abundant charged particles. More details can be found in our published work $[10,11]$.

\section{Results}

The analysis to extract the freeze-out parameters $T$ and $\mu_{B}$ on lower moments of net-protons and netcharge has been performed in a wide range of beam energy $\sqrt{s_{N N}}=11.5,19.6,27,39,62.4,200 \mathrm{GeV}$ for the most central collisions $(0-5 \%)$. The simultaneous comparison of the susceptibility ratios $\chi_{2} / \chi_{1}$ for net-protons and net-charge allow us to obtain a freeze-out parametrization within a narrow range of variation for both temperature and baryo-chemical potential.

In Fig. 1 we show the results for the ratio $\chi_{2} / \chi_{1}$ for both net-proton and net-charge evaluated in our HRG model as a function of the collision energy, along with experimental data from $[16,17]$. The combined fit of these lower moment ratios to data permits to obtain a freeze-out parametrization which describes very well both the net-proton and the net-charge observables.

The results for the temperature and baryo-chemical potential at chemical freeze-out in our approach are presented in Fig. 2 and compared to the one obtained in [3].

The values obtained for the temperature are lower than those given in [3] and the ones extracted from fits to particle yields and ratios [1,26]. A possible explanation for such a difference could be that the analysis performed here is mainly driven by non-strange particles, namely pions and protons. Recent fits to data from ALICE and STAR collaborations show a tension between the so-called light particles $(p, \pi)$ and hyperons $(\Sigma, \Xi, \Omega)$ when trying to reproduce the yields with one value of $T$, namely it seems that hyperons demand a higher freeze-out temperature. LQCD studies of the deconfinement transition from quarks and gluons to hadrons, also show a similar flavour hierarchy [27]. In [10] we reproduce quite well the STAR data on particle ratios for $\sqrt{s}=200 \mathrm{GeV}$, but hyperons are underestimated for our value of $T_{c h}$. However the discussion on the so-called proton anomaly is still ongoing.

The freeze-out parameters we obtained show nevertheless a nice agreement with a similar study on fluctuations performed on the lattice by the Wuppertal-Budapest collaboration [8]. 


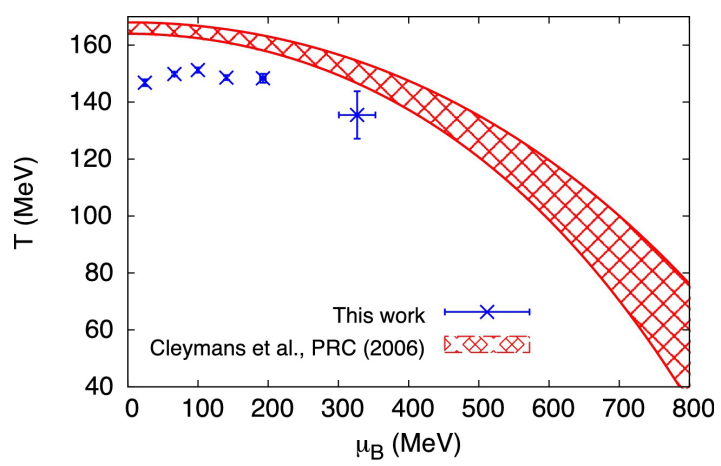

Figure 2: Freeze-out parametrization in $\left(T, \mu_{B}\right)$ plane obtained in this work and results from Cleymans et al. [3].

Using our freeze-out conditions we evaluated the higher-order susceptibility ratios for both netproton and net-electric charge. Results are presented in Fig. 3.
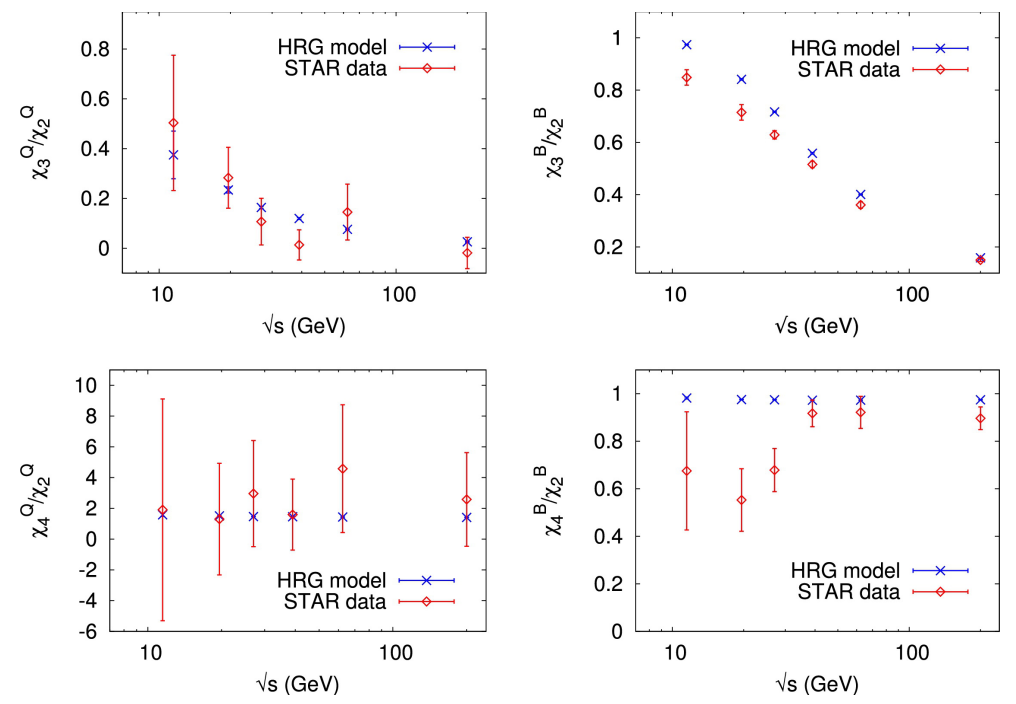

Figure 3: HRG model calculations with our freeze-out parametrization for $\chi_{3} / \chi_{2}$ and $\chi_{4} / \chi_{2}$ for net-electric (left panels) and net-baryon charge (right panels).

The net-electric charge ratios are well described by our freeze-out conditions, as can be seen from the left side of the plot, while a discrepancy between our calculations and data on net-baryon is evident, especially at lower beam energies. This disagreement might come from an overestimate of KA corrections at lower energies. Moreover we cannot reproduce the dip in the $\chi_{4}^{B} / \chi_{2}^{B}$ ratio at low energies, but the description of data improves as $\sqrt{s}$ increases. Still, these inconsistencies between the HRG calculation and data might derive from an onset of chiral critical behaviour at finite $\mu_{B}$, mainly in the third and fourth order cumulants [28, 29].

The values for the freeze-out temperature and baryo-chemical potential are listed in Table 1. 
Table 1: Freeze-out values for $T$ and $\mu_{B}$ at several energies obtained in our HRG model.

\begin{tabular}{c|cc}
\hline$\sqrt{s}(\mathrm{GeV})$ & $T(\mathrm{MeV})$ & $\mu_{B}(\mathrm{MeV})$ \\
\hline 11.5 & $135.5 \pm 8.3$ & $326.7 \pm 25.9$ \\
19.6 & $148.4 \pm 1.6$ & $192.5 \pm 3.9$ \\
27 & $148.5 \pm 0.7$ & $140.4 \pm 1.4$ \\
39 & $151.2 \pm 0.8$ & $99.9 \pm 1.4$ \\
62.4 & $149.9 \pm 0.5$ & $66.4 \pm 0.6$ \\
200 & $146.8 \pm 1.2$ & $24.3 \pm 0.6$ \\
\hline
\end{tabular}

\section{Conclusions}

We presented a study on ratios of lower-order fluctuations for net-protons and net-electric charge in a HRG approach compared to data from the STAR collaboration in order to extract the chemical freeze-out parameters $T$ and $\mu_{B}$. Experimental acceptance cuts and isospin randomization through the KA formalism have been taken into account in our calculations. In particular this analysis shows that it is possible to describe both net-electric and net-proton lower moments in a wide range of collision energies and that these observables represent a useful tool to determine the freeze-out conditions.

The small uncertainties on the data of the moments of multiplicity distributions from STAR collaboration allow to constrain the freeze-out temperatures in a range $\leq 5 \mathrm{MeV}$. Within this parametrization, higher-order moments for net-charge are well reproduced while there are discrepancies at low beam energy for the net-proton observables, probably due to an overestimate of the KA corrections and to the onset of a chiral critical behaviour in higher cumulants such as $\chi_{3}$ and $\chi_{4}$. The freeze-out conditions obtained in our approach are lower with respect to previous analyses coming from fits to particle yields and ratios but our results show agreement with similar studies in LQCD. A possible way to explain the disagreement with the values of $T$ extracted from yields, containing both strange and non-strange hadrons, is to assume that particles without a strangeness content, i.e. protons and pions, which drive the net-charge and net-proton fluctuations, require a lower freeze-out $T$.

\section{Acknowledgements}

This work is supported by the FIRB Research Grant RBFR0814TT, DAAD and the DOE grants DEFG02-03ER41260, DEFG02-05ER41367 and DE-FG02-07ER41521.

\section{References}

[1] A. Andronic, P. Braun-Munzinger, J. Stachel, Nucl.Phys. A772, 167 (2006), nucl-th/0511071

[2] F. Becattini, J. Manninen, M. Gazdzicki, Phys.Rev. C73, 044905 (2006), hep-ph/0511092

[3] J. Cleymans, H. Oeschler, K. Redlich, S. Wheaton, Phys.Rev. C73, 034905 (2006), hep-ph/0511094

[4] J. Manninen, F. Becattini, Phys.Rev. C78, 054901 (2008), 0806.4100

[5] A. Andronic, P. Braun-Munzinger, K. Redlich, J. Stachel, J.Phys. G38, 124081 (2011), 1106.6321

[6] F. Karsch, Central Eur.J.Phys. 10, 1234 (2012), 1202 . 4173 
[7] S. Borsanyi, Z. Fodor, S. Katz, S. Krieg, C. Ratti et al., Phys.Rev.Lett. 111, 062005 (2013), 1305.5161

[8] S. Borsanyi, Z. Fodor, S. Katz, S. Krieg, C. Ratti et al., Phys.Rev.Lett. 113, 052301 (2014), 1403.4576

[9] A. Bazavov, H. Ding, P. Hegde, O. Kaczmarek, F. Karsch et al., Phys.Rev.Lett. 109, 192302 (2012), 1208.1220

[10] P. Alba, W. Alberico, R. Bellwied, M. Bluhm, V. Mantovani Sarti et al., Phys.Lett. B738, 305 (2014), 1403.4903

[11] M. Bluhm, P. Alba, W. Alberico, R. Bellwied, V. Mantovani Sarti et al., Nucl.Phys. A (2014), 1408.4734

[12] V. Begun, M.I. Gorenstein, M. Hauer, V. Konchakovski, O. Zozulya, Phys.Rev. C74, 044903 (2006), nucl-th/0606036

[13] F. Karsch, K. Redlich, Phys.Lett. B695, 136 (2011), 1007. 2581

[14] J. Fu, Phys.Lett. B722, 144 (2013)

[15] P. Garg, D. Mishra, P. Netrakanti, B. Mohanty, A. Mohanty et al., Phys.Lett. B726, 691 (2013), 1304.7133

[16] L. Adamczyk et al. (STAR Collaboration), Phys.Rev.Lett. 112, 032302 (2014), 1309. 5681

[17] L. Adamczyk et al. (STAR Collaboration), Phys.Rev.Lett. 113, 092301 (2014), 1402.1558

[18] S. Borsanyi, Z. Fodor, C. Hoelbling, S.D. Katz, S. Krieg et al., Phys.Lett. B730, 99 (2014), 1309.5258

[19] A. Bazavov et al. (HotQCD Collaboration), Phys.Rev. D90, 094503 (2014), 1407.6387

[20] H. Bebie, P. Gerber, J. Goity, H. Leutwyler, Nucl.Phys. B378, 95 (1992)

[21] J. Beringer et al. (Particle Data Group), Phys.Rev. D86, 010001 (2012)

[22] M. Bluhm, P. Alba, W. Alberico, A. Beraudo, C. Ratti, Nucl.Phys. A929, 157 (2014), 1306.6188

[23] M. Kitazawa, M. Asakawa, Phys.Rev. C85, 021901 (2012), 1107. 2755

[24] M. Kitazawa, M. Asakawa, Phys.Rev. C86, 024904 (2012), 1205. 3292

[25] M. Nahrgang, M. Bluhm, P. Alba, R. Bellwied, C. Ratti (2014), 1402 . 1238

[26] A. Andronic, P. Braun-Munzinger, J. Stachel, Acta Phys.Polon. B40, 1005 (2009), 0901. 2909

[27] R. Bellwied, S. Borsanyi, Z. Fodor, S.D. Katz, C. Ratti, Phys.Rev.Lett. 111, 202302 (2013), 1305.6297

[28] K. Redlich, Central Eur.J.Phys. 10, 1254 (2012), 1207.2610

[29] V. Skokov, B. Friman, K. Redlich, Phys.Lett. B708, 179 (2012), 1108.3231 
